\title{
Projective modules over polynomial rings
}

\author{
by Shizuo ENDO
}

(Received April 20, 1963)

Introduction. In this paper a ring means a commutative ring with a unit element. An integral domain $R$ is said to be a PF domain if any finite projective $R$-module is free.

J.P. Serre raised in [6] the following question:

(S) Is the polynomial ring $K\left[X_{1}, X_{2}, \cdots, X_{n}\right]$ with a finite number of variables $X_{1}, X_{2}, \cdots, X_{n}$ over a field $K$ a PF domain?

It is well-known (cf. [3]) that any principal ideal domain is a PF domain. Therefore, when $n=1$, the answer to $(\mathrm{S})$ is affirmative. Also, when $n=2$, C.S. Seshadri gave in [9] an affirmative answer to (S). He proved in [9], more generally, that the polynomial ring $R[X]$ with a variable $X$ over a principal ideal domain $R$ is a PF domain. This was further generalized by Seshadri [10], Serre [8] and Bass [1] to the following form: If $R^{\prime}=R[X]$ is the polynomial ring with a variable $X$ over a Dedekind domain $R$, then every finite projective $R^{\prime}$-module $P^{\prime}$ is expressible as $P^{\prime} \cong R^{\prime} \bigotimes_{R} P$ for some finite projective $R$-module $P$.

For $n \geqq 3$ the question ( $\mathrm{S}$ ) is still open. D. Lissner gave in [4] some results suggesting a negative answer to (S) in case $n \geqq 3$. Recently $\mathrm{H}$. Bass and S. Schanuel showed in [2] that, for the polynomial ring $R^{\prime}=R\left[X_{1}, X_{2}\right.$, $\left.\cdots, X_{n}\right]$ with a finite number of variables $X_{1}, X_{2}, \cdots, X_{n}$ over a semi-local principal ideal domain $R$, any projective $R^{\prime}$-module of rank $>n$ is free.

Our main results in this paper are Theorems 4.7 and 5.4. In Theorem 4.7 we give a necessary and sufficient condition for a Noetherian integral domain $R$ of dimension 1, whose derived normal ring (cf. [5]) is a finite $R$-module, to the effect that, for the polynomial ring $R^{\prime}=R[X]$ with a variable $X$ over $R$, every finite projective $R^{\prime}$-module $P^{\prime}$ is expressible as $P^{\prime} \cong R^{\prime} \bigotimes_{R} P$ for some finite projective $R$-module $P$. This is a generalization of all the above-mentioned result in [1], [8], [9] and [10]. In Theorem 5.4, we give a necessary and sufficient condition for a semi-local integral domain $R$ of dimension 1 to the effect that the polynomial ring $R[X, Y]$ with two variables $X, Y$ over $R$ is a PF domain. This is related with the above-mentioned result in [2].

In both cases, the conditions are expressed in the form that the ring $R$ should be "weakly normal". Thus the concept of weakly normal rings will 
play an important part throughout this paper. This concept will be introduced in $\S 1$. Another fundamental tool of this paper is the Proposition 2.2 in $\S 2$, which seems to be the most general result proved by the method used by Seshadri, Serre and Bass.

Our notations and terminologies are the same as those in [5] for the ringtheoretical facts with some exceptions. The "dimension" of a ring means the "altitude" of it in [5]. An ideal a of a ring $R$ is said to be "unmixed" in it if, for any prime divisor $\mathfrak{p}$ of $\mathfrak{a}$ in $R$, we have height ${ }_{R} \mathfrak{p}=$ height $_{R} \mathfrak{a}$. A "multiplicative system" of a ring means a multiplicatively closed subset of it which does not contain 0 . Let $R$ be a ring, $S$ be a multiplicative system of $R$ and $M$ be an $R$-module. Then we denote by $R_{S}$ and $M_{S}$ the quotient ring and the quotient module of $R$ and $M$ with respect to $S$, respectively. Especially, if $S$ is the complementary set of a prime ideal $\mathfrak{p}$ in $R$, we use $R_{\mathfrak{p}}$ and $M_{p}$ instead of $R_{S}$ and $M_{S}$, respectively. A projective $R$-module $P$ is said to be of "rank $n$ " in $R$ if, for any maximal ideal $\mathfrak{m}$ of $R, P_{\mathfrak{m}}$ has an $R_{\mathfrak{m}}$-free base consisting of $n$ elements. A projective $R$-module is called "quasi-free" if it is expressible as a direct sum of $R$-modules, each of which is isomorphic to an ideal generated by an idempotent of $R$. Let $R^{\prime}=R\left[X_{1}, X_{2}, \cdots, X_{n}\right]$ be the polynomial ring with a finite number of variables $X_{1}, X_{2}, \cdots, X_{n}$ over a ring $R$. Then a finite $R^{\prime}$-module $M^{\prime}$ is said to be "extended" if there exists a finite $R$-module $M$ such that $M^{\prime} \cong R^{\prime} \bigotimes_{R} M$.

\section{$\S 1$. Preliminaries.}

First we refer to some well-known facts, which will be freely used throughout this paper.

LEMMA 1.1 (cf. [3]). Let $R$ be an integral domain and $M$ be a finite $R$ module. Then $M$ is $R$-projective if and only if, for any maximal ideal $\mathfrak{m}$ of $R$, $M_{\mathrm{m}}$ is $R_{\mathrm{m}}$-free.

Lemma 1.2 (cf. [7]). A semi-local integral domain is a PF domain.

LeMma 1.3 (cf. [5]). Let $R$ be an integral domain and $\bar{R}$ be an integral extension of $R$. Then, for any prime ideal $\mathfrak{p}$ of $R$, there is a prime ideal $\bar{p}$ of $\bar{R}$ such that $\mathfrak{p}=\overline{\mathfrak{p}} \cap R$. Furthermore, $\overline{\mathfrak{p}}$ has height 1 in $\bar{R}$ if $\mathfrak{p}$ has height 1 in $R$, and $\mathfrak{p}$ is maximal in $R$ if and only if $\bar{p}$ is maximal in $\bar{R}$.

LEMMA 1.4 (cf. [5]). Let $R$ be a Noetherian integral domain of dimension 1 and $\bar{R}$ be an almost finite integral extension of $R$. Then $\bar{R}$ is also a Noetherian integral domain of dimension 1 , and, if $\bar{R}$ is a normal ring, it is a Dedekind domain. Especially, if $R$ is semi-local, then $\bar{R}$ is also semi-local, and, in case $\bar{R}$ is normal, it is a principal ideal domain.

Lemma 1.5 (cf. [5]). Let $R$ be a Noetherian ring and $R^{\prime}=R\left[X_{1}, X_{2}, \cdots, X_{n}\right]$ 
be the polynomial ring with a finite number of variables $X_{1}, X_{2}, \cdots, X_{n}$ over $R$. Then there exists a maximal ideal $\mathfrak{m}^{\prime}$ in $R^{\prime}$ such that $\mathfrak{m}^{\prime} \cap R$ is not maximal in $R$ if and only if there is a prime ideal $\mathfrak{p}$ in $R$ such that $R / \mathfrak{p}$ is a semi-local integral domain of dimension 1 . If $\mathfrak{m}^{\prime} \cap R$ is not maximal in $R, R / \mathfrak{m}^{\prime} \cap R$ becomes, in fact, a semi-local integral domain of dimension 1.

We now introduce a notion of weakly normal rings.

DEFINITION. Let $R$ be a local integral domain of dimension 1 with a maximal ideal $\mathfrak{m}$. Let $\bar{R}$ be the derived normal ring of $R$ and $\overline{\mathfrak{n}}$ be the Jacobson radical of $\bar{R}$. Then $R$ is said to be a weak (discrete) valuation ring if we have $\mathfrak{m}=\overline{\mathfrak{n}}$ in the set-theoretical sense.

In general, a Noetherian integral domain $R$ is said to be a weakly normal ring if $R$ satisfies the following two conditions:

1) For any prime ideal $\mathfrak{p}$ of height 1 in $R, R_{\mathfrak{p}}$ is a weak valuation ring.

2) Any principal ideal $(\neq 0)$ in $R$ is unmixed, i. e., any prime divisor of it has height 1 in $R$.

Now we prove

PROPOSITION 1.6. A Noetherian normal ring is a weakly normal ring. However, a weakly normal ring is not always a normal ring.

ProOF. The first part of our proposition is obvious by our definition (cf. [5]). Hence we have only to give an example of a weak valuation ring which is not a valuation ring. Let $Q$ be the field of all rational numbers and $Q[[X, Y]]$ be the formal power-series ring with two variables $X, Y$ over $\dot{Q}$. If we put $\mathfrak{p}=\left(X^{2}+Y^{2}\right) Q[[X, Y]]$, then $\mathfrak{p}$ is a prime ideal of $Q[[X, Y]]$. Furthermore put $R_{0}=Q[[X, Y]] / \mathfrak{p}$ and denote by $a, b$ the residues of $X, Y$ in $R_{0}$, respectively. Then $R_{0}$ is a local integral domain of dimension 1 with a maximal ideal $\mathfrak{m}_{0}=a R_{0}+b R_{0}$. Let $\bar{R}$ be the derived normal ring of $R_{0}$. As $R_{0}$ is complete, $\bar{R}$ is a valuation ring with a maximal ideal $\overline{\mathfrak{m}}$ (cf. [5]). Let $R$ be an integral domain generated by $\overline{\mathfrak{m}}$ over $R_{0}$. Then $R$ is obviously a weak valuation ring. If we put $\alpha=b / a$, then we have $\alpha^{2}+1=0$, hence $\alpha, \alpha^{-1} \in \bar{R}$, and so $\alpha \in \overline{\mathfrak{m}}$. If we suppose $\alpha \in R$, then we have $\alpha=u+\beta$ for a unit $u$ of $R_{0}$ and an element $\beta$ of $\overline{\mathfrak{m}}$. From $\alpha^{2}+1=0$, we obtain $\beta(\beta+2 u)+u^{2}$ $+1=0$. As $\beta \in \overline{\mathfrak{m}}$, we have $u^{2}+1 \in \overline{\mathfrak{m}} \cap R_{0}=\mathfrak{m}_{0}$. Let $f(X, Y)$ be a representative of $u$ in $Q[[X, Y]]$. Then we have $(f(X, Y))^{2}+1 \in X Q[[X, Y]]+Y Q[[X$, $Y]$. If we denoted by $q$ the constant term of $f(X, Y)$, then we obtain $q^{2}+1=0$. As $q \in Q$, this is obviously a contradiction. Thus $\alpha \notin R$. This shows $R \neq \bar{R}$. Consequently $R$ is a weak valuation ring which is not a valuation ring.

Here we show some basic properties of weakly normal rings.

LEMMA 1.7. Let $R$ be a weakly normal ring and $S$ be a multiplicative system of $R$. Then $R_{S}$ is also a weakly normal ring. 
Lemma 1.8. Let $R$ be a Noetherian integral domain of dimension 1. Then $R$ is a weakly normal ring if and only if, for any prime ideal $\mathfrak{p}$ of $R, R_{\mathfrak{p}}$ is a weak valuation ring. Especially, if $R$ is semi-local, and $\mathfrak{n}$ is the Jacobson radical of $R$, then $R$ is a weakly normal ring if and only if we have $\mathfrak{n}=\overline{\mathfrak{n}}$ for the Jacobson radical $\bar{n}$ of the derived normal ring of $R$.

These two results follow immediately from our definition.

LEMMA 1.9. Let $R$ be a weakly normal ring. Then the polynomial ring $R^{\prime}=R\left[X_{1}, X_{2}, \cdots, X_{n}\right]$ with a finite number of variables $X_{1}, X_{2}, \cdots, X_{n}$ over $R$ is also a weakly normal ring.

PROOF. Let us show our lemma for $n=1$, i. e., $R^{\prime}=R[X]$ with a variable $X$. The general case follows easily by induction on $n$. First we prove that, for any prime ideal $\mathfrak{p}^{\prime}$ of height 1 in $R^{\prime}, R_{\mathfrak{p}^{\prime}}^{\prime}$ is a weak valuation ring. If $\mathfrak{p}^{\prime} \cap R=0$, then we have $R_{\mathfrak{p}^{\prime}}^{\prime}=(K[X])_{\mathfrak{p}^{\prime} K[X]}$ where $K$ is the quotient field of $R$, and so $R_{\mathfrak{p}^{\prime}}^{\prime}$ is a discrete valuation ring. On the other hand, if $\mathfrak{p}^{\prime} \cap R=\mathfrak{p} \neq 0$, then we have $\mathfrak{p}^{\prime}=\mathfrak{p} R^{\prime}$, hence $R_{\mathfrak{p}^{\prime}}^{\prime}=\left(R_{\mathfrak{p}}[X]\right)_{\mathfrak{p} R \mathfrak{p}[X]}$. Therefore we may suppose that $R$ is a weak valuation ring and $\mathfrak{p}$ is a maximal ideal of it. Let $\bar{R}$ be the derived normal ring of $R$ and $\bar{n}$ be the Jacobson radical of $\bar{R}$. Then we have $p=\bar{n}$. Since $\bar{R}[X]$ is the derived normal ring of $R[X]$, putting $S^{\prime}=R[X]-\mathfrak{p} R[X],(\bar{R}[X])_{S^{\prime}}$ is also the derived normal ring of $(R[X])_{\mathfrak{p} R[X]}$. From $\mathfrak{p}=\bar{n}$ we see easily $\mathfrak{p}(R[X])_{p R[X]}=\bar{n}(\bar{R}[X])_{S^{\prime}}$. As $\bar{n}(\bar{R}[X])_{S^{\prime}}$ coincides with the Jacobson radical of $(\bar{R}[X])_{S^{\prime}}$, this shows that $(R[X])_{\mathfrak{p} R[X]}$ is a weak valuation ring. Thus, in every case, $R_{p^{\prime}}^{\prime}$ is a weak valuation ring.

Let $\mathfrak{a}^{\prime}$ be a non-zero principal ideal of $R^{\prime}$ and $\mathfrak{p}^{\prime}$ be a prime divisor of it. Suppose height ${ }_{R^{\prime}} \mathfrak{p}^{\prime} \geqq 2$. Then we have $\mathfrak{p}^{\prime} \cap R \neq 0$. Hence there exists a non-zero element $a$ of $R$ in $\mathfrak{p}^{\prime}$. Then $\mathfrak{p}^{\prime}$ is a prime divisor of $\alpha R^{\prime}$ (cf. [5], 12.6). However, as $R$ is weakly normal, $a R$ is unmixed in $R$, and so $a R^{\prime}$ is unmixed in $R^{\prime}$. This is a contradiction. Thus $\mathfrak{p}^{\prime}$ must be of height 1 in $R^{\prime}$, i. e., $\mathfrak{a}^{\prime}$ must be unmixed in $R^{\prime}$. This completes our proof.

\section{$\S 2$. Fundamental proposition.}

We first prove a proposition, which gives in the most general form a reasoning which played essential parts in the proofs of the Seshadri-SerreBass's results.

LEMMA 2.1. Let $R$ be an integral domain and $\mathfrak{p}$ be a prime ideal of $R$ such that $R / p$ is Euclidean. Let $P$ be a finite projective $R$-module and $L$ be a finite projective $R$-submodule of $P$ such that $L=L_{1} \oplus L_{2} \oplus \cdots \oplus L_{n}$ with each $L_{i} a$ finite projective $R$-module of rank 1 . Then there exists an automorphism $\varphi$ of $L$ and an integer $r, 0 \leqq r \leqq n$, such that $\varphi\left(L_{1} \oplus L_{2} \oplus \cdots \oplus L_{r}\right) \subset \mathfrak{p} P$ and $\varphi\left(L_{r+1}\right.$ $\left.\oplus L_{r+2} \oplus \cdots \oplus L_{n}\right) \cap \mathfrak{p} P=\mathfrak{p} \cdot \varphi\left(L_{r+1} \oplus L_{r+2} \oplus \cdots \oplus L_{n}\right)$.

The proof is omitted, because it runs in the same line as in [1], 2.2. 
Proposition 2.2. Let $R$ be a Noetherian integral domain and $S$ be a multiplicative system of $R$ consisting of elements $\left\{a_{i}\right\}$ such that $a_{i} R$ is expressible as the product of invertible prime ideals $\mathfrak{p}_{1}, \mathfrak{p}_{2}, \cdots, \mathfrak{p}_{s}$ in $R$, for any $\mathfrak{p}_{i}$ of which $R / p_{i}$ is Euclidean. Let $P$ be a finite projective $R$-module. If $P_{S}$ is $R_{S^{-}}$ free, then $P$ is expressible as a direct sum of projective $R$-modules of rank 1. Especially, if $S$ is a multiplicative system of $R$ generated by prime elements $\left\{p_{i}\right\}$ such that each $R / p_{i} R$ is Euclidean, and if $P_{S}$ is $R_{S}$-free, then $P$ is $R$-free.

Proof (cf. [1], [8], [9] or [10]). As $P_{S}$ is $R_{S}$-free, we can choose a free base $u_{1}, u_{2}, \cdots, u_{t}$ for $P_{S}$ in $P$. If we put $L=u_{1} R+u_{2} R+\cdots+u_{t} R$, then we have $L \subset P$ and $s P \subset L$ for some $s \in S$. By our assumption on $S$ we have $s R=\mathfrak{p}_{1}^{r_{1}} \mathfrak{p}_{2}^{r_{2}} \cdots \mathfrak{p}_{s}^{r_{s}}$ for invertible prime ideals $\mathfrak{p}_{1}, \mathfrak{p}_{2}, \cdots, \mathfrak{p}_{s}$ in $R$ such that all $R / \mathfrak{p}_{i}$ 's are Euclidean. Hence we established the existence of an $R$-submodule $L$ of $P$ satisfying the following conditions:

1) $L=L_{1} \oplus L_{2} \oplus \cdots \oplus L_{n}$ with each $L_{i}$ a projective $R$-module of rank 1 .

2) $\mathfrak{p}_{1}^{l_{1}} \mathfrak{p}_{2}^{l_{2}} \cdots \mathfrak{p}_{k}^{l_{k}} P \subset L$ with each $\mathfrak{p}_{i}$ an invertible prime ideal of $R$ such that $R / \mathfrak{p}_{i}$ is Euclidean.

Since $R$ is Noetherian, we may choose $L$ maximal satisfying 1). Now we shall prove $P=L$. Suppose $L \subsetneq P$. If we put $\mathfrak{p}=\mathfrak{p}_{1}$ and $\mathfrak{a}=\mathfrak{p}_{1}^{l_{1}-1} \mathfrak{p}_{2}^{l_{2}} \cdots \mathfrak{p}_{k}^{l_{k}}\left(l_{1} \geqq 1\right)$, we may assume $\mathfrak{p a} P \subset L$ but $\mathfrak{a} P \subset L$. If $\mathfrak{p a} P \subset \mathfrak{p} L$, then $\mathfrak{a} P \subset \mathfrak{p}^{-1} \mathfrak{p} L=L$, contradiction. Therefore $\mathfrak{p a} P \subset \mathfrak{p} L$, however $\mathfrak{p} a P \subset \mathfrak{p} P \cap L$. This shows that $\mathfrak{p} L \subsetneq \mathfrak{p} P \cap L$.

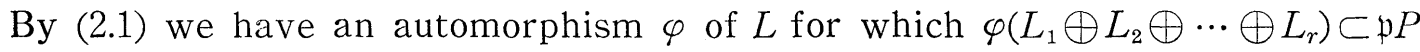
and $\mathfrak{p} P \cap \varphi\left(L_{r+1} \oplus L_{r+2} \oplus \cdots \oplus L_{n}\right)=\mathfrak{p} \cdot \varphi\left(L_{r+1} \oplus L_{r+2} \oplus \cdots \oplus L_{n}\right)$. As $\mathfrak{p} P \cap L \neq \mathfrak{p} L$, we have $r>0$. Hence, if we put $H=\mathfrak{p}^{-1} \cdot \varphi\left(L_{1} \oplus L_{2} \oplus \cdots \oplus L_{r}\right) \oplus \varphi\left(L_{r+1} \oplus L_{r+2} \oplus\right.$ $\cdots \oplus L_{n}$ ), then $H$ contradicts the maximality of $L$. Thus we obtain $P=L$. This completes the proof of the first part of our proposition. The second part is obvious from the above proof of the first part.

COROllary 2.3. Let $R$ be a Noetherian integral domain and $S$ be a multiplicative system of $R$ consisting of elements $\left\{a_{i}\right\}$ such that each $a_{i} R$ is expressible as the product of maximal invertible ideals in $R$. Let $R^{\prime}=R[X]$ be the polynomial ring with a variable $X$ over $R$ and $P^{\prime}$ be a finite projective $R^{\prime}$-module. If $P_{S}^{\prime}$ is $R_{S}^{\prime}$-free, then $P^{\prime}$ is expressible as a direct sum of projective $R^{\prime}$-modules of rank 1. Especially, if $S$ is a multiplicative system of $R$ generated by prime elements $\left\{p_{i}\right\}$ such that all $p_{i} R^{\prime}$ s are maximal in $R$ and if $P_{S}^{\prime}$ is $R_{S^{\prime}}$-free, then $P^{\prime}$ is also $R^{\prime}$-free.

LEMMA 2.4. Let $R$ be a ring and $P$ be a finite quasi-free $R$-module. If $P$ has its rank, then $P$ is a free $R$-module.

ProOF. Put $n=\operatorname{rank}_{R} P$. When $n=0$, this is obvious. Hence we may assume $n \geqq 1$. We put $P=e_{1} R \oplus e_{2} R \oplus \cdots \oplus e_{t} R$ for idempotents, $e_{1}, e_{2}, \cdots, e_{t}$ $(\mathrm{t} \geqq n)$ in $R$. If we put $e_{2}^{\prime}=\left(1-e_{1}\right) e_{2}, e_{3}^{\prime}=\left(1-e_{1}\right)\left(1-e_{2}\right) e_{3}, \cdots, e_{t}^{\prime}=\left(1-e_{1}\right)\left(1-e_{2}\right)$ $\cdots\left(1-e_{t-1}\right) e_{t}$ and $e_{2}^{\prime \prime}=e_{2}-e_{2}^{\prime}, \cdots, e_{t}^{\prime \prime}=e_{t}-e_{t}^{\prime}$, then we have $P \cong e_{1} R \oplus e_{2}^{\prime} R \oplus \cdots$ 
$\oplus e_{t}^{\prime} R \oplus e_{2}^{\prime \prime} R \oplus e_{3}^{\prime \prime} R \oplus \cdots \oplus e_{t}^{\prime \prime} R$. We see easily that $R \cong e_{1} R \oplus e_{2}^{\prime} R \oplus \cdots \oplus e_{t}^{\prime} R$. Putting $P^{\prime}=e_{2}^{\prime \prime} R \oplus e_{3}^{\prime \prime} R \oplus \cdots \oplus e_{t}^{\prime \prime} R$, then $P^{\prime}$ is a quasi-free $R$-module of rank $n-1$ and we have $P \cong R \oplus P^{\prime}$. By repeating this procedure to $P^{\prime}$, we conclude that $P$ is a free $R$-module of rank $n$.

Proposition 2.5. Let $R$ be an integral domain, $\mathfrak{n}$ be an ideal contained in the Jacobson radical of $R$ and $P$ be a finite projective $R$-module. If $P / \mathfrak{n} P$ is a quasi-free $R / \mathfrak{n}$-module, then $P$ is $R$-free.

Proof. As $R$ is an integral domain, $P$ has its rank. If we put $n=\operatorname{rank}_{R} P, P_{\mathrm{m}}$ is a free $R_{\mathfrak{m}}$-module of rank $n$, for any maximal ideal $\mathrm{m}$ of $R$. Since $\mathfrak{n} \subset \mathfrak{m},(P / \mathfrak{n} P)_{\mathfrak{m} / \mathfrak{n}}$ is also a free $(R / \mathfrak{n})_{\mathfrak{m} / \mathfrak{n}}$-module of rank $n$. Therefore we have $\operatorname{rank}_{R / \mathfrak{n}} P / \mathfrak{n} P=n$. As $P / \mathfrak{n} P$ is $R / \mathfrak{n}$-quasi-free, $P / \mathfrak{n} P$ is $R / \mathfrak{n}$-free by (2.4). Let $\left\{\bar{u}_{1}, \bar{u}_{2}, \cdots, \bar{u}_{n}\right\}$ be a free base of $P / \mathfrak{n} P$ over $R / \mathfrak{n} R$, and $u_{1}, u_{2}, \cdots, u_{n}$ be the representatives of $\bar{u}_{1}, \bar{u}_{2}, \cdots, \bar{u}_{n}$ in $P$, respectively. If we put $M=u_{1} R+u_{2} R+$ $\cdots+u_{n} R$, we have $P=M+\mathfrak{n} P$. Then, by the Krull-Azumaya's lemma, we obtain $P=M$, i. e., $P$ has a base $\left\{u_{1}, u_{2}, \cdots, u_{n}\right\}$ over $R$. As $P_{\mathrm{m}}$ is of rank $n$, $\left\{u_{1}, u_{2}, \cdots, u_{n}\right\}$ is a free base of $P_{\mathfrak{m}}$ over $R_{\mathfrak{m}}$ for any maximal ideal $\mathfrak{m}$ of $R$. From this it follows that $\left\{u_{1}, u_{2}, \cdots, u_{n}\right\}$ is a free base of $P$ over $R$. Thus $P$ is $R$-free.

\section{$\S 3$. Polynomial rings over semi-local integral domains of dimension $1, \mathrm{I}$.}

Here we give

Proposition 3.1. Let $R$ be a semi-local integral domain of dimension 1 with the Jacobson radical $\mathfrak{n}$ and $R^{\prime}=R\left[X_{1}, X_{2}, \cdots, X_{n}\right]$ be the polynomial ring with a finite number of variables $X_{1}, X_{2}, \cdots, X_{n}$ over $R$. Then the following conditions are equivalent:

1) $R$ is a weakly normal ring.

2) Any prime ideal $\mathfrak{p}^{\prime}$ of height 1 in $R^{\prime}$ such that $\mathfrak{p}^{\prime}+\mathfrak{n} R^{\prime}=R^{\prime}$ is principal in $R^{\prime}$.

Proof. Let $\bar{R}$ be the derived normal ring of $R$ and $\bar{n}$ be the Jacobson radical of $\bar{R}$. Denote by $K$ the quotient field of $R$ and put $\bar{R}^{\prime}=\bar{R}\left[X_{1}, X_{2}, \cdots, X_{n}\right]$.

The implication 1$) \rightarrow 2$ ): Suppose that $R$ is weakly normal. Then, by (1.8), we have $n=\bar{n}$, hence $n R^{\prime}=\bar{n} \bar{R}^{\prime}$. Let $\mathfrak{p}^{\prime}$ be a prime ideal of height 1 in $R^{\prime}$ such that $\mathfrak{p}^{\prime}+\mathfrak{n} R^{\prime}=R^{\prime}$. As $\bar{R}^{\prime}$ is integral over $R^{\prime}$, there exists a prime ideal $\overline{\mathfrak{p}}^{\prime}$ of height 1 in $\bar{R}^{\prime}$ such that $\mathfrak{p}^{\prime}=\overline{\mathfrak{p}}^{\prime} \cap R^{\prime}$ by (1.3). Since $\bar{R}$ is a semilocal principal ideal domain by (1.4), $\bar{R}^{\prime}$ is a unique factorization domain, as is well-known, hence $\overline{\mathfrak{p}}^{\prime}$ is principal in $\bar{R}^{\prime}$ (cf. [5], 13.1). As $\overline{\mathfrak{p}}^{\prime}+\overline{\mathfrak{n}} \bar{R}^{\prime}=\bar{R}^{\prime}$, we have $\overline{\mathfrak{p}}^{\prime}=(f+1) \bar{R}^{\prime}$ for some $f \in \overline{\mathfrak{n}} \bar{R}^{\prime}=\mathfrak{n} R^{\prime}$. From $\mathfrak{p}^{\prime}=\overline{\mathfrak{p}}^{\prime} \cap R^{\prime}$, we obtain $f+1 \in \mathfrak{p}^{\prime}$. Hence we have also $\mathfrak{p}^{\prime} K\left[X_{1}, X_{2}, \cdots, X_{n}\right]=(f+1) K\left[X_{1}, X_{2}, \cdots, X_{n}\right]=\overline{\mathfrak{p}}^{\prime} K\left[X_{1}, X_{2}\right.$, $\left.\cdots, X_{n}\right]$. If $\mathfrak{m}^{\prime}$ is a maximal ideal of $R^{\prime}$ containing $f+1$, then we have $\mathfrak{m}^{\prime} \cap R=0$ 
and so we have $R_{\mathfrak{m}^{\prime}}^{\prime}=\left(K\left[X_{1}, X_{2}, \cdots, X_{n}\right]\right)_{\mathfrak{m}^{\prime} K\left[X_{1}, \cdots, X_{n}\right]}$. Therefore we have $\mathfrak{p}^{\prime} R_{\mathfrak{m}^{\prime}}^{\prime}=(f+1) R_{\mathfrak{m}}^{\prime}$. Thus we must have $\mathfrak{p}^{\prime}=(f+1) R^{\prime}$. This proves that $\mathfrak{p}^{\prime}$ is principal in $R^{\prime}$.

The implication 2) $\rightarrow 1$ ): Suppose that $R$ is not weakly normal. Then, again by (1.8), we have a) $\mathfrak{n} \neq \bar{R}$ or b) $\mathfrak{n} \bar{R}=\mathfrak{n} \neq \overline{\mathfrak{n}}$. In order to prove 2$) \rightarrow 1$ ) it suffices to show that there exists a non-principal prime ideal $\mathfrak{p}^{\prime}$ of height 1 in $R^{\prime}$ such that $\mathfrak{p}^{\prime}+\mathfrak{n} R^{\prime}=R^{\prime}$, in case a), b).

Case a): When $\mathfrak{n} \bar{R} \neq \mathfrak{n}$, we see easily $\mathfrak{n} \bar{R} \leftarrow R$. Therefore we find an element $b / a$ of $\bar{R}, a, b \in R$ such that $b c / a \notin R$ for a suitable element $c$ of $\mathfrak{n}$. As $b / a \notin R$ but $b / a \in \bar{R}, b / a$ is the root of an equation:

$$
T^{k}+r_{1} T^{k-1}+\cdots+r_{k-1} T+r_{k}=0, \quad r_{1}, r_{2}, \cdots, r_{k} \in R, \quad k \geqq 2 .
$$

Hence $b c / a$ is also the root of an eqation:

$$
T^{k}+c r_{1} T^{k-1}+\cdots+c^{k-1} r_{k-1} T+c^{k} r_{k}=0 .
$$

If we put $d_{i}=c^{i} r_{i}, 1 \leqq i \leqq k$, then all $d_{i}$ 's are contained in $\mathfrak{n}$. Hence $a / b c$ is the root of the following equation:

$$
d_{k} T^{k}+d_{k-1} T^{k-1}+\cdots d_{1} T+1=0 .
$$

On the other hand, as is easily seen, $a / b c$ is also the root of an equation $b c T-a=0 . \quad$ Put $\mathfrak{p}^{\prime}=\left(b c X_{1}-a\right) R^{\prime}+\left(d_{k} X_{1}^{k}+d_{k-1} X_{1}^{k-1}+\cdots+d_{1} X_{1}+1\right) R^{\prime}$. We shall now prove that $\mathfrak{p}^{\prime}$ is as required. Let $\mathfrak{m}^{\prime}$ be a maximal ideal of $R^{\prime}$. If $\mathfrak{m}^{\prime} \supset \mathfrak{p}^{\prime}$, we have $\mathfrak{m}^{\prime} \cap R=0$, as $d_{1}, d_{2}, \cdots, d_{k} \in \mathfrak{n}$, hence we obtain $R_{\mathfrak{m}^{\prime}}^{\prime}=\left(K\left[X_{1}\right.\right.$, $\left.\left.X_{2}, \cdots, X_{n}\right]\right)_{\mathfrak{m}^{\prime} K\left[X_{1}, \cdots, X_{n}\right]}$. Therefore we have $\mathfrak{p}^{\prime} R_{\mathfrak{m}^{\prime}}^{\prime}=\left(X_{1}-a / b c\right) R_{\mathfrak{m} \prime}^{\prime}$. If $\mathfrak{m}^{\prime} D \mathfrak{p}^{\prime}$, we have $\mathfrak{p}^{\prime} R_{\mathfrak{m}}^{\prime}=R_{\mathfrak{m}}^{\prime}$. This shows that $\mathfrak{p}^{\prime}$ is an invertible prime ideal of height 1 in $R^{\prime}$. However, as $b c \notin a R, \mathfrak{p}^{\prime}$ is not principal in $R^{\prime}$, and, as $d_{1}, d_{2}, \cdots, d_{k} \in \mathfrak{n}$, we have $\mathfrak{p}^{\prime}+\mathfrak{n} R^{\prime}=R^{\prime}$. This shows that $\mathfrak{p}^{\prime}$ is as required.

Case b): As $\mathfrak{n} \bar{R}=\mathfrak{n} \neq \overline{\mathfrak{n}}$, we find a positive integer $k \geqq 2$ such that $\overline{\mathfrak{n}}^{k} \subset \mathfrak{n}$. Then there exists an element $b / a$ of $\overline{\mathfrak{n}}$ such that $b / a \notin \mathfrak{n}$ but $(b / a)^{k}=c \in \mathfrak{n}$. Hence $a / b$ is the root of equations $c T^{k}-1=0$ and $b T-a=0$. If we put $\mathfrak{p}^{\prime}=\left(b X_{1}-a\right) R^{\prime}+\left(c X_{1}^{k}-1\right) R^{\prime}$, we can prove, as in case a), that $\mathfrak{p}^{\prime}$ is as required. This completes our proof.

COROllary 3.2. Let $R$ be a semi-local integral domain of dimension 1 and $R^{\prime}=R\left[X_{1}, X_{2}, \cdots, X_{n}\right]$ be the polynomial ring with a finite number of variables $X_{1}, X_{2}, \cdots, X_{n}$ over $R$. If $R$ is not weakly normal, then there exists an invertible ideal in $R^{\prime}$ which is not principal.

PROOF. It is obvious from the proof of 2$) \rightarrow 1$ ) in (3.1).

PROPOSITION 3.3. Let $R$ be a semi-local integral domain of dimension 1 and let $R^{\prime}=R[X]$ be the polynomial ring with a variable $X$ over $R$. Then the following conditions are equivalent:

1) $R$ is a weakly normal ring. 
2) $R^{\prime}$ is a PF domain.

Proof. The implication 2$) \rightarrow 1$ ) was already proved by (3.2). Hence we have only to show the implication 1$) \rightarrow 2$ ). Suppose that $R$ is weakly normal. Let $\mathfrak{n}$ be the Jacobson radical of $R$, and $\mathfrak{m}^{\prime}$ be a maximal ideal of height 1 in $R^{\prime}$. Then we have $\mathfrak{m}^{\prime}+\mathfrak{n} R^{\prime}=R^{\prime}$ by (1.5). Therefore, by (3.1), $\mathfrak{m}^{\prime}$ is principal in $R^{\prime}$. Let $S^{\prime}$ be the multiplicative system of $R^{\prime}$ generated by all prime generators of maximal ideals of height 1 in $R^{\prime}$. Then, for any maximal ideal $\mathfrak{m}^{\prime}$ of height 2 in $R^{\prime}$, we have $\mathfrak{m}^{\prime} \cap S^{\prime}=\phi$. Accordingly, if we put $R^{\prime \prime}=R_{S^{\prime}}^{\prime}$, $\mathfrak{m}^{\prime} R^{\prime \prime}$ is also maximal in $R^{\prime \prime}$. Conversely, for any maximal ideal $\mathfrak{m}^{\prime \prime}$ of $R^{\prime \prime}$, $\mathfrak{m}^{\prime \prime} \cap R^{\prime}$ is a maximal ideal of height 2 in $R^{\prime}$. As any maximal ideal of height 2 in $R^{\prime}$ contains $n R^{\prime}$, any maximal ideal of $R^{\prime \prime}$ contains $n R^{\prime \prime}$ and so $" R^{\prime \prime}$ is contained in the Jacobson radical of $R^{\prime \prime}$. Now we have $R^{\prime \prime} / \mathrm{n} R^{\prime \prime}$ $\cong(R / \mathfrak{n})[X] \cong\left(R / \mathfrak{m}_{1}\right)[X] \oplus \cdots \oplus\left(R / \mathfrak{m}_{t}\right)[X]$, where $\mathfrak{m}_{1}, \mathfrak{m}_{2}, \cdots, \mathfrak{m}_{t}$ are all maximal ideals of $R$. Since any $\left(R / \mathfrak{m}_{i}\right)[X]$ is a PF domain, any projective $R^{\prime \prime} / \mathfrak{n} R^{\prime \prime}$. module is quasi-free. Hence, by (2.5), $R^{\prime \prime}$ is a PF domain. By applying (2.2) to $S^{\prime}$, we see that $R^{\prime}$ is also a PF domain.

\section{$\S 4$. Polynomial rings over Noetherian integral domains of dimension 1.}

First we give two lemmas.

LEMMA 4.1. Let $R$ be an integral domain and $R^{\prime}=R[X]$ be the polynomial ring with a variable $X$ over $R$. Then any finite projective $R^{\prime}$-module of rank 1 is isomorphic to an invertible ideal of $R^{\prime}$ containing a non-zero element of $R$.

PROOF. Let $P^{\prime}$ be a finite projective $R^{\prime}$-module of rank 1 . If we denote by $K$ the quotient field of $R$, we have $P^{\prime} \subset K \bigotimes_{R} P^{\prime}$. As $K \bigotimes_{R} P^{\prime}$ is a free $K[X]$-module of rank 1 , we may choose a generator $\alpha$ of $K \bigotimes_{R} P^{\prime}$ such that $P^{\prime} \subset \alpha R^{\prime}$. If we consider $\alpha R^{\prime}$ as $R^{\prime}$, we can also consider $P^{\prime}$ as an invertible ideal $\mathfrak{a}^{\prime}$ of $R^{\prime}$. Since, for some $a \neq 0$ in $R$, we have $a \alpha \in P^{\prime}$, we have clearly $\mathfrak{a}^{\prime} \cap R \neq 0$.

LEMMA 4.2. Let $R$ be a $\mathrm{PF}$ domain and $R^{\prime}=R[X]$ be the polynomial ring with a variable $X$ over $R$. Let $P^{\prime}$ be a finite projective $R^{\prime}$-module of rank 1 . If $P^{\prime}$ is extended, then $P^{\prime}$ is isomorphic to $R^{\prime}$.

Proof. As this is easy, we omit it (cf. [3], X, Ex. 1).

Now we prove

THeORem 4.3. Let $R$ be a Noetherian integral domain such that any principal ideal in it is unmixed, and $R^{\prime}=R\left[X_{1}, X_{2}, \cdots, X_{n}\right]$ be the polynomial ring with a finite number of variables $X_{1}, X_{2}, \cdots, X_{n}$ over $R$. Then the following conditions are equivalent:

1) $R$ is a weakly normal ring. 
2) Any finite projective $R^{\prime}$-module of rank 1 is extended.

PROOF. It suffices to prove our theorem in case $n=1$, i. e., $R^{\prime}=R[X]$ with a variable $X$, by the last part of the proof of (1.9) and by (1.9).

The implication 1$) \rightarrow 2$ ): Suppose that $R$ is weakly normal. According to (4.1), it suffices to show that, for an invertible ideal $\mathfrak{a}^{\prime}$ of $R^{\prime}$ such that $\mathfrak{a}^{\prime} \cap R$ $=\mathfrak{a} \neq 0$, we have $\mathfrak{a}^{\prime}=\mathfrak{a} R^{\prime}$. As, for any maximal ideal $\mathfrak{m}^{\prime}$ of $R^{\prime}, \mathfrak{a}^{\prime} R_{\mathfrak{m}}^{\prime}$, is principal in $R_{\mathfrak{m}}^{\prime}, \mathfrak{a}^{\prime} R_{\mathfrak{m}}^{\prime}$, is unmixed in $R_{\mathfrak{m}}^{\prime}$, by (1.7) and (1.9). So $\mathfrak{a}^{\prime}$ must be unmixed in $R^{\prime}$. Let $\mathfrak{p}_{1}^{\prime}, \mathfrak{p}_{2}^{\prime}, \cdots, \mathfrak{p}_{t}^{\prime}$ be all prime divisors of $\mathfrak{a}^{\prime}$ in $R^{\prime}$. As all $\mathfrak{p}_{i}^{\prime}$ s contain $\mathfrak{a}$, we have $\mathfrak{p}_{i}^{\prime} \cap R=\mathfrak{p}_{i} \neq 0$ for any $i$. As height ${ }_{R^{\prime}} \mathfrak{p}_{i}^{\prime}=1$ we have $\mathfrak{p}_{i}^{\prime}=\mathfrak{p}_{i} R^{\prime}$ for any $i$. Now let $\mathfrak{a}^{\prime}=\mathfrak{q}_{1}^{\prime} \cap \mathfrak{q}_{2}^{\prime} \cap \cdots \cap \mathfrak{q}_{t}^{\prime}$ be a primary decomposition of $\mathfrak{a}^{\prime}$ in $R^{\prime}$ where each $\mathfrak{q}_{i}^{\prime}$ is a primary ideal belonging to $\mathfrak{p}_{i} R^{\prime}$. Then we have also $\mathfrak{q}_{i}^{\prime} \cap R=\mathfrak{q}_{i} \neq 0$, for any $i$. Now we shall prove $\mathfrak{q}_{i} R^{\prime}=\mathfrak{q}_{i}^{\prime}$ for any $i$. Since $R_{\mathfrak{p}_{i}}$ is a weak valuation ring, $R_{\mathfrak{p}_{i}}[X]$ is a PF domain by (3.3). As we have $\mathfrak{a}^{\prime} R_{\mathfrak{p}_{i}}[X]=\mathfrak{q}_{i}^{\prime} R_{\mathfrak{p}_{i}}[X]$, and $\mathfrak{a}^{\prime} R_{\mathfrak{p}_{i}}[X]$ is invertible in $R_{\mathfrak{p}_{i}}[X], \mathfrak{q}_{i}^{\prime} R_{\mathfrak{p}_{i}}[X]$ is principal in $R_{\mathfrak{p}_{i}}[X]$. From $\mathfrak{q}_{i} \neq 0 \mathfrak{q}_{i}^{\prime} R_{\mathfrak{p}_{i}}[X]$ has a generator $q_{i}$ in $R_{\mathfrak{p}_{i}}$. If we put $q_{i}^{*}=q_{i} R_{p_{i}}$, we have $q_{i}^{*} R_{p_{i}}[X]=\mathfrak{q}_{i}^{\prime} R_{p_{i}}[X]$ and $c_{i}^{\prime} R_{p_{i}}[X] \cap R_{p_{i}}=q_{i}^{*}$. Therefore $\mathfrak{q}_{i}^{*}$ is a primary ideal belonging to $\mathfrak{p}_{i} R_{\mathfrak{p}_{i}}$. Since $\mathfrak{q}_{i}=\mathfrak{q}_{i}^{*} \cap R$, we have $\mathfrak{q}_{i} R_{\mathfrak{p}_{i}}=\mathfrak{q}_{i}^{*}$, and so $\mathfrak{q}_{i}$ is also a primary ideal belonging to $\mathfrak{p}_{i}$. As $\mathfrak{q}_{i} R_{\mathfrak{p}_{i}}[X]$ $=\mathfrak{q}_{i}^{\prime} R_{\mathfrak{p}}[X]$, we have $\mathfrak{q}_{i} R^{\prime}=\mathfrak{q}_{i}^{\prime}$. From this we easily see $\mathfrak{a}=\mathfrak{q}_{1} \cap \mathfrak{q}_{2} \cap \cdots \cap \mathfrak{q}_{t}$ and $\mathfrak{a}^{\prime}=\mathfrak{a} R^{\prime}$. This proves 1$) \rightarrow 2$ ).

The implication 2$) \rightarrow 1$ ): Suppose that $R$ is not weakly normal. Then, for some prime ideal $\mathfrak{p}$ of height 1 in $R, R_{\mathfrak{p}}$ is not a weak valuation ring. Let $\bar{R}$ be the derived normal ring of $R$, and put $S=R-p$. Then $\bar{R}_{S}$ is the derived normal ring of $R_{\mathfrak{p}}$. As $R_{\mathfrak{p}}$ is not a weak valuation ring, $\mathfrak{p} R_{\mathfrak{p}}$ does not coincide with the Jacobson radical $\bar{n}$ of $\bar{R}_{S}$ in the set-theoretical sense. Hence we have a) $\mathfrak{p} \bar{R}_{S} \neq \mathfrak{p} R_{\mathfrak{p}}$ or b) $\mathfrak{p} \bar{R}_{S}=\mathfrak{p} R_{\mathfrak{p}} \neq \overline{\mathfrak{n}}$. It suffices to show that there is an invertible ideal in $R^{\prime}$ which is not extended, in each case a), b).

Case a): There exists an element $\beta$ of $\bar{R}_{S}$ such that $c \beta \notin R_{\mathfrak{p}}$ for some $c \in \mathfrak{p} R_{\mathfrak{p}}$. By multiplying $\beta, c$ by a suitable element of $S$, we may assume $\beta \in \bar{R}$ such that $c \beta \in R_{\mathfrak{p}}$ for some $c \in \mathfrak{p}$. Put $\beta=b / a, a, b \in R$. Then there exist only a finite number of prime divisors $\mathfrak{p}_{1}(=\mathfrak{p}), \mathfrak{p}_{2}, \cdots, \mathfrak{p}_{t}$ of $a R$, each of which is of height 1 in $R$. If we put $U=\bigcap_{i=1}^{t}\left(R-\mathfrak{p}_{i}\right), R_{U}$ is a semi-local integral domain of dimension 1 with maximal ideals $\mathfrak{p}_{1} R_{U}, \mathfrak{p}_{2} R_{U}, \cdots, \mathfrak{p}_{t} R_{U}$, which is not weakly normal. Again, by multiplying $c$ by a suitable element of $\bigcap_{i=2}^{t} \mathfrak{p}_{i}$ which is not contained in $\mathfrak{p}$, we may assume that $\beta$ is an element of $\bar{R}$ such that $c \beta \notin R_{U}$ for some $c \in \bigcap_{i=1}^{t} \mathfrak{p}_{i}$. If we put $\alpha=c \beta$, then, as in (3.3), $\alpha^{-1}$ is a root of an equation $c f(T)+1=0, f(T) \in R[T]$. Furthermore put $\mathfrak{p}^{\prime}=(b c X$ $-a) R^{\prime}+(c f(X)+1) R^{\prime}$. Let $\mathfrak{m}^{\prime}$ be a maximal ideal of $R^{\prime}$. If we put $q=\mathfrak{m}^{\prime} \cap R$, 
$\mathfrak{q}$ is a prime ideal of $R$. If $c \in \mathfrak{q}$, then $c f(X)+1 \oplus \mathfrak{m}^{\prime}$, hence we have $\mathfrak{p}^{\prime} R_{\mathfrak{m}}^{\prime}=R_{\mathfrak{m}}^{\prime}$ 。 If $c \notin \mathfrak{q}$, then $\mathfrak{p}_{1}, \mathfrak{p}_{2}, \cdots, \mathfrak{p}_{t} \leftarrow \mathfrak{q}$, and so $a \notin \mathfrak{q}$, i. e., $a \notin \mathfrak{m}^{\prime}$. Therefore we have $\mathfrak{p}^{\prime} R_{\mathfrak{m}}^{\prime}=(\alpha X-1) R_{\mathfrak{m}}^{\prime}$. This shows that $\mathfrak{p}^{\prime}$ is an invertible prime ideal of $R^{\prime}$. However, $\mathfrak{p}^{\prime} R_{U}[X]$ is not principal in $R_{U}[X]$ as in the proof of Case a) in (3.3). By (4.2), then, $\mathfrak{p}^{\prime} R_{U}[X]$ is not extended in $R_{U}[X]$. Consequently $\mathfrak{p}^{\prime}$ is not extended in $R^{\prime}$. Thus $\mathfrak{p}^{\prime}$ is as required.

Case b): There exists an element $\alpha$ of $\bar{R}_{S}$ such that $\alpha \notin R_{\mathfrak{p}}$ but $\alpha^{k} \in \mathfrak{p} R_{\mathfrak{p}}$ for some $k \geqq 2$. As in a) we may assume $\alpha \in \bar{R}$ such that $\alpha \notin R_{\mathfrak{p}}$ but $\alpha^{k} \in \mathfrak{p} R_{\mathfrak{p}}$. Put $\alpha=b / a, a, b \in R$. There exist a finite number of prime divisors $\mathfrak{p}_{1}(=\mathfrak{p}), \mathfrak{p}_{2}, \cdots, \mathfrak{p}_{t}$ of $a R$. If we put $U=\bigcap_{i=1}^{t}\left(R-\mathfrak{p}_{i}\right)$, then we may assume also that $\alpha$ is an element of $\bar{R}$ such that $\alpha \notin R_{U}$ and $\alpha^{k} \in \bigcap_{i=1}^{t} \mathfrak{p}_{i}$. If we put $c=\alpha^{k}$ and $\mathfrak{p}^{\prime}=(b X-a) R^{\prime}+\left(c X^{k}-1\right) R^{\prime}$, then, as in a), we can show that $\mathfrak{p}^{\prime}$ is as required. This completes our proof.

COROLlary 4.4. Let $R$ be a Noetherian integral domain of dimension 1 and $R^{\prime}=R\left[X_{1}, X_{2}, \cdots, X_{n}\right]$ be the polynomial ring with a finite number of variables $X_{1}, X_{2}, \cdots, X_{n}$ over $R$. Then the following conditions are equivalent:

1) $R$ is a weakly normal ring.

2) Any finite projective $R$-module of rank 1 is extended.

COROLlaRY 4.5 (cf. [1]). Let $R$ be a Noetherian normal ring and $R^{\prime}$ $=R\left[X_{1}, X_{2}, \cdots, X_{n}\right]$ be the polynomial ring with a finite number of variables $X_{1}, X_{2}, \cdots, X_{n}$ over $R$. Then any finite projective $R^{\prime}$-module of rank 1 is extended.

LEMMA 4.6. Let $R$ be a Noetherian integral domain, whose derived normal ring, $\bar{R}$, is a finite $R$-module. Then there exist only a finite number of prime ideals $\mathfrak{p}_{1}, \mathfrak{p}_{2}, \cdots, \mathfrak{p}_{t}$ of height 1 in $R$ such that $R_{\mathfrak{p}_{i}}$ 's are not discrete valuation rings.

Proof. Let $\mathfrak{a}$ be the conductor of $\bar{R}$ to $R$, i. e., $\mathfrak{a}=\{a ; a \bar{R} \subset R, a \in R\}$. By our assumption $\mathfrak{a}$ is a non-zero ideal of $R$. There exist only a finite number of prime ideals $\mathfrak{p}_{1}, \mathfrak{p}_{2}, \cdots, \mathfrak{p}_{t}$ of height 1 in $R$ containing $\mathfrak{a}$. If $\mathfrak{p}$ is a prime ideal of height 1 in $R$ which does not coincide with any of $\mathfrak{p}_{i}$ 's, we have $\bar{R} \subset R_{\mathfrak{p}}$, as $a \bar{R} \subset R$. Putting $S=R-\mathfrak{p}$, we have $R_{\mathfrak{p}}=\bar{R}_{S}$. This shows that $R_{\mathfrak{p}}$ is a discrete valuation ring.

Our main result is given in the following:

THEOREM 4.7. Let $R$ be a Noetherian integral domain of dimension 1, whose derived normal ring, $\bar{R}$, is a finite $R$-module and let $R^{\prime}=R[X]$ be the polynomial ring with a variable $X$ over $R$. Then the following conditions are equivalent:

1) $R$ is a weakly normal ring.

2) Any finite projective $R^{\prime}$-module is extended. 
Proof. The implication 2) $\rightarrow 1$ ) was proved in (4.3). Hence we have only to show the implication 1$) \rightarrow 2$ ). As $R$ is one-dimensional, any prime ideal $\mathfrak{p}(\neq 0)$ of $R$ is of height 1 in $R$. According to (4.6), there exists only a finite number of prime ideals $\mathfrak{p}_{1}, \mathfrak{p}_{2}, \cdots, \mathfrak{p}_{t}$ in $R$ such tnat $R_{\mathfrak{p}_{i}}$ 's are not discrete valuation rings. Let $\mathfrak{p}$ be a prime ideal of $R$ different from $\mathfrak{p}_{1}, \mathfrak{p}_{2}, \cdots, \mathfrak{p}_{t}$. Then $R_{\mathfrak{p}}$ is a discrete valuation ring and so $\mathfrak{p}$ is invertible in $R$. Now put $S=\bigcap_{i=1}^{t}\left(R-\mathfrak{p}_{i}\right)$. Then, for any element $s$ of $S$, we have $s \notin \mathfrak{p}_{1}, \mathfrak{p}_{2}, \cdots, \mathfrak{p}_{t}$, hence $s R$ is expressible as the product of invertible prime ideals of $R$. Therefore $S$ satisfies the condition in (2.3). Suppose that $R$ is a weakly normal ring. Then $R_{S}$ is also weakly normal. Hence, by (3.3), $R_{S}^{\prime}=R_{S}[X]$ is a PF domain. So, by (2.3), any finite projective $R^{\prime}$-module is expressible as a direct sum of projective $R^{\prime}$-modules of rank 1 . On the other hand, by (4.4), any finite projective $R^{\prime}$-module of rank 1 is extended. Thus our proof is completed.

COROLlARY 4.8. Let $R$ be a Noetherian PF integral domain of dimension 1 , whose derived normal ring, $\bar{R}$, is a finite $R$-module, and let $R^{\prime}=R[X]$ be the polynomial ring with a variable $X$ over $R$. Then the following conditions are equivalent:

1) $R$ is a weakly normal ring.

2) $R^{\prime}$ is a PF domain.

§ 5. Polynomial rings over semi-local integral domains of dimension 1 , II.

We begin with

LemMA 5.1. Let $R$ be a unique factorization domain and $R^{\prime}=R\left[X_{1}, X_{2}, \cdots, X_{n}\right]$ be the polynomial ring with a finite number of variables $X_{1}, X_{2}, \cdots, X_{n}$ over $R$. Let $\mathfrak{a}^{\prime}$ be an ideal of $R^{\prime}$ generated by $a_{1} X_{1}+b_{1}, a_{2} X_{2}+b_{2}, \cdots, a_{n} X_{n}+b_{n}$ such that, for any $i, a_{i}, b_{i}$ are relatively prime non-zero elements of $R$. Then there exist relatively prime non-zero elements $a_{i}, b_{i}$ of $R$ and positive integers $l_{2}, \cdots, l_{n}$. such that $a\left(X_{1}+X_{2}^{l_{2}}+\cdots+X_{n}^{l_{n}}\right)+b \in \mathfrak{a}^{\prime}$ and $a \in \bigcap_{i=1}^{n} a_{i} R$.

Proof. This is obvious in case $n=1$. First we suppose $n=2$. If $a_{1}, a_{2}$ are relatively prime, $a_{1} a_{2}$ and $a_{2} b_{1}+a_{1} b_{2}$ are also relatively prime. We have now $a_{1}\left(a_{2} X_{2}+b_{2}\right)+a_{2}\left(a_{1} X_{1}+b_{1}\right)=a_{1} a_{2}\left(X_{1}+X_{2}\right)+a_{2} b_{1}+a_{1} b_{2} \in \mathfrak{a}^{\prime}$. Hence, if we put $a=a_{1} a_{2}, b=a_{1} b_{2}+a_{2} b_{1}$ and $l_{2}=1$, then our conclusion holds. If $a_{1}, a_{2}$ are not relatively prime, then there exist common prime divisors $p_{1}, p_{2}, \cdots, p_{t}$ of $a_{1}, a_{2}$ and we may write $a_{1}=p_{1}^{r_{1}} p_{2}^{r_{2}} \cdots p_{t}^{r_{t}} a_{1}^{\prime}$ and $a_{2}=p_{1}^{s_{1}} p_{2}^{s_{2}} \cdots p_{t}^{s_{t}} a_{2}^{\prime}$ for relatively prime elements $a_{1}^{\prime}, a_{2}^{\prime}$ of $R$ such that $a_{1}^{\prime}, a_{2}^{\prime} \notin p_{i} R, 1 \leqq i \leqq t$ and positive integers $r_{i}, s_{i}$. Now, for a suitably large integer $l$, we have $l s_{i}>r_{i}$ for any $i$ and $a_{2}^{l} X_{2}^{l}+b_{2}^{l} \in a^{\prime}$. Then we have

$$
\begin{aligned}
& p_{1}^{l s_{1}-r_{1}} p_{2}^{l s_{2}-r_{2}} \cdots p_{t}^{l s_{t}-r_{t}} a_{2}^{\prime l}\left(a_{1} X_{1}+b_{1}\right)+a_{1}^{\prime}\left(a_{2}^{l} X_{2}^{l}+b_{2}^{l}\right) \\
= & p_{1}^{l_{1}} p_{2}^{l s_{2}} \cdots p_{t}^{l s_{t}} a_{1}^{\prime} a_{2}^{\prime l}\left(X_{1}+X_{2}^{l}\right)+p_{1}^{l s_{1}-r_{1}} p_{2}^{l s_{2}-r_{2}} \cdots p_{t}^{l s_{t}-r_{t}} a_{2}^{\prime l} b_{1}+a_{1}^{\prime} b_{2}^{l} \in \mathfrak{a}^{\prime} .
\end{aligned}
$$


If we put $a=p_{1}^{l s_{1}} p_{2}^{l s_{2}} \cdots p_{t}^{l s_{t}}$ and $b=p_{1}^{l s_{1}-r_{1}} p_{2}^{l s_{2}-r_{2}} \cdots p_{t}^{l s_{t}-r_{t}} a_{2}^{\prime l} b_{1}+a_{1}^{\prime} b_{2}^{l}$, then $a, b$ are relatively prime non-zero elements of $R$. Accordingly, for $a, b, l_{2}=l$, our conclusion holds.

In general case, we use the induction on $n$. Suppose that the conclusion holds for $n=k(\geqq 2)$. Let $\mathfrak{a}_{k+1}^{\prime}$ be an ideal of $R\left[X_{1}, X_{2}, \cdots, X_{k}, X_{k+1}\right]$ generated by $a_{1} X_{1}+b_{1}, a_{2} X_{2}+b_{2}, \cdots, a_{k} X_{k}+b_{k}, a_{k+1} X_{k+1}+b_{k+1}$ such that for any $i, a_{i}, b_{i}$, are relatively prime non-zero elements of $R$. If we put $\mathfrak{a}_{k}^{\prime}=\left(a_{1} X_{1}+b_{1}\right) R\left[X_{1}, X_{2}\right.$, $\left.\cdots, X_{k}\right]+\cdots+\left(a_{k} X_{k}+b_{k}\right) R\left[X_{1}, X_{2}, \cdots, X_{k}\right]$, then $\mathfrak{a}_{k}^{\prime}$ is also an ideal of $R\left[X_{1}, X_{2}\right.$, $\left.\cdots, X_{k}\right]$ satisfying the condition in our lemma for $n=k$. By the assumption of induction, there is an element $a^{\prime}\left(X_{1}+X_{2}^{l_{2}}+\cdots+X_{k}^{l_{k}}\right)+b^{\prime}$ in $\mathfrak{a}_{k}^{\prime}$ such that $a^{\prime} \in \bigcap_{i=1}^{k} a_{i} R$ and $a^{\prime} R+b^{\prime} R=R, a^{\prime}, b^{\prime} \neq 0 . \quad$ As $\mathfrak{a}_{k}^{\prime} \subset \mathfrak{a}_{k+1}^{\prime}, a^{\prime}\left(X_{1}+X_{2}^{l_{2}}+\cdots+X_{k}^{l k}\right)+b^{\prime}$ $\in \mathfrak{a}_{k+1}^{\prime}$. Put $Y=X_{1}+X_{2}^{l_{2}}+\cdots+X_{k}^{l_{k}}$ and let $\mathfrak{a}^{\prime \prime}$ be an ideal of $R\left[Y, X_{k+1}\right]$ generated by $a^{\prime} Y+b^{\prime}, a_{k+1} X_{k+1}+b_{k+1}$. If we apply the proof for $n=2$ to $a^{\prime} Y+b^{\prime}, a_{k+1} X_{k+1}+b_{k+1}$, we can find relatively prime elements $a, b$ of $R$ such that $a\left(Y+X_{k+1}^{l_{k+1}}\right)+b \in \mathfrak{a}^{\prime \prime}$ and $a \in a^{\prime} R \cap a_{k+1} R$. As $\mathfrak{a}^{\prime \prime} \subset \mathfrak{a}_{k+1}^{\prime}$, we have $a\left(Y+X_{k+1}^{l_{k+1}}\right)+b=a\left(X_{1}+X_{2}^{l_{2}}+\cdots+X_{k+1}^{l_{k+1}}\right)+b \in \mathfrak{a}_{k+1}^{\prime}$. Obviously we also have $a \in \bigcap_{i=1}^{k+1} a_{i} R$. This shows that our conclusion holds for $n=k+1$. Thus our proof is completed.

Proposition 5.2. Let $R$ be a semi-local integral domain of dimension 1 and $R^{\prime}=R\left[X_{1}, X_{2}, \cdots, X_{n}\right]$ be the polynomial ring with a finite number of variables $X_{1}, X_{2}, \cdots, X_{n}$ over $R$. Then any maximal ideal $\mathfrak{m}^{\prime}$ of height $n$ in $R^{\prime}$ contains a prime ideal $\mathfrak{p}^{\prime}$ of height 1 in $R^{\prime}$ such that $R^{\prime} / \mathfrak{p}^{\prime}$ is isomorphic to the polynomial ring with $n-1$ variables over a field.

Proof. If $n=1$, this is trivial. Hence we may suppose $n \geqq 2$. As $\mathfrak{m}^{\prime}$ has height $n$ in $R^{\prime}$, at least one of $X_{i}$ 's is not contained in $\mathfrak{m}^{\prime}$. Suppose that $X_{i_{1}}, X_{i_{2}}, \cdots, X_{i_{k}} \notin \mathfrak{m}^{\prime}$ and $X_{j_{1}}, X_{j_{2}}, \cdots, X_{j_{n-k}} \in \mathfrak{m}^{\prime}$, for some $k \geqq 1$. Then we have $\mathfrak{m}^{\prime}=\mathfrak{m}^{\prime \prime} R^{\prime}+X_{j_{1}} R^{\prime}+X_{j_{2}} R^{\prime}+\cdots+X_{j_{n-k}} R^{\prime}$ for a maximal ideal $\mathfrak{m}^{\prime \prime}$ of height $k$ in $R\left[X_{i_{1}}, X_{i_{2}}, \cdots, X_{i_{k}}\right]$.

If we use $\mathfrak{m}^{\prime \prime}$ instead of $\mathfrak{m}^{\prime}$, we may assume $X_{1}, X_{2}, \cdots, X_{n} \notin \mathfrak{m}^{\prime}$. If we put $\mathfrak{p}_{i}=\mathfrak{m}^{\prime} \cap R\left[X_{i}\right]$ for any $i$, then $\mathfrak{p}_{i}$ is a prime ideal of height 1 in $R\left[X_{i}\right]$ and we have $\mathfrak{p}_{i} \cap R=0$. If at least one $\mathfrak{p}_{i_{0}}$ of $\mathfrak{p}_{i}$ 's is maximal in $R\left[X_{i_{0}}\right]$, then we have $R^{\prime} / \mathfrak{p}_{i_{0}} R^{\prime}=\left(R\left[X_{i_{0}}\right] / \mathfrak{p}_{i_{0}}\right)\left[X_{1}, X_{2}, \cdots, X_{i_{0}-1}, X_{i_{0}+1}, \cdots, X_{n}\right]$, and therefore our assertion is true. Hence we assume that any $\mathfrak{p}_{i}$ is not maximal in $R\left[X_{i}\right]$. Let $K$ be the quotient field of $R$. Then $\mathfrak{p}_{i} K\left[X_{i}\right]$ is a prime ideal of $K\left[X_{i}\right]$, and so it is generated by an irreducible polynomial $f_{i}\left(X_{i}\right)$ in $K\left[X_{i}\right]$. As is easily seen, we can suppose $f_{i}\left(X_{i}\right) \in \mathfrak{p}_{i}$. Let $\alpha_{1}^{(i)}, \alpha_{2}^{(i)}, \cdots, \alpha_{t_{i}}^{(i)}$ be all the roots of the algebraic equation $f_{i}(T)=0$ over $K$ for any $i$, and put $\bar{K}=K\left[\alpha_{1}^{(1)}, \alpha_{2}^{(1)}\right.$, $\left.\cdots, \alpha_{t_{1}}^{(1)}, \alpha_{1}^{(2)}, \cdots, \alpha_{1}^{(n)}, \cdots, \alpha_{t_{n}}^{(n)}\right]$. Let $\bar{R}$ be the integral closure of $R$ in $\bar{K}$. Then, by (1.4), $\bar{R}$ is a semi-local principal ideal domain. If we put $\bar{R}^{\prime}=\bar{R}\left[X_{1}, X_{2}\right.$, 
$\left.\cdots, X_{n}\right]$, then $\bar{R}^{\prime}$ is a unique factorization domain integral over $R^{\prime}$. There exists a maximal ideal $\overline{\mathfrak{m}}^{\prime}$ of $\bar{R}^{\prime}$ such that $\mathfrak{m}^{\prime}=\overline{\mathfrak{m}}^{\prime} \cap R^{\prime}$ by (1.3). Similarly, by (1.3), we have height $\bar{R}_{\bar{\prime}^{\prime}} \overline{\mathrm{m}}^{\prime}=n$. Since any $f_{i}\left(X_{i}\right)$ is expressible as the product of linear polynomials in $\bar{R}\left[X_{i}\right]$, and any $X_{i}$ is not contained in $\overline{\mathfrak{m}}^{\prime}$, we have

$$
\overline{\mathfrak{m}}^{\prime}=\left(\bar{a}_{1} X_{1}+\bar{b}_{1}\right) \bar{R}^{\prime}+\left(\bar{a}_{2} X_{2}+\bar{b}_{2}\right) \bar{R}^{\prime}+\cdots+\left(\bar{a}_{n} X_{n}+\bar{b}_{n}\right) \bar{R}^{\prime},
$$

for non-zero relatively prime elements $\bar{a}_{i}, \bar{b}_{i}$ of $\bar{R}$ for any $i$. Then, by (5.1), there is an element $\bar{a}\left(X_{1}+X_{2}^{l_{2}}+\cdots+X_{n}^{l_{n}}\right)+\bar{b}$ in $\overline{\mathfrak{m}}^{\prime}$ such that $\bar{a} \bar{R}+\bar{b} \bar{R}=\bar{R}$ and $\bar{a} \in \bigcap_{i=1}^{n} \bar{a}_{i} \bar{R}$. As $\overline{\mathfrak{m}}^{\prime}$ is maximal in $\bar{R}^{\prime}$, it is easily seen that $\prod_{i=1}^{n} \bar{a}_{i}$ is contained in the Jacobson radical $\overline{\mathfrak{n}}$ of $\bar{R}$. Hence $\bar{a}$ is also contained in $\overline{\mathfrak{r}}$. Since $\bar{a} \bar{R}+\bar{b} \bar{R}=\bar{R}, \bar{b}$ must be a unit of $\bar{R}$, and so we may suppose $\bar{b}=1$. If we put $U=X_{1}+X_{2}^{l_{2}}+\cdots+X_{n}^{l_{n}}$, then we have $R^{\prime}=R\left[U, X_{2}, \cdots, X_{n}\right]$ and $\bar{R}^{\prime}=\bar{R}\left[U, X_{2}\right.$, $\left.\cdots, X_{n}\right]$. Also we have $\overline{\mathfrak{m}}^{\prime} \cap \bar{R}[U]=(\bar{a} U+1) \bar{R}[U]$. As $\bar{a} \in \overline{\mathfrak{m}}$, $(\bar{a} U+1) \bar{R}[U]$ is a maximal ideal of height 1 in $\bar{R}[U]$. If we put $\mathfrak{p}_{U}=(\bar{a} U+1) \bar{R}[U] \cap R[U]$, then $\mathfrak{p}_{U}$ is a prime ideal of height 1 in $R[U]$. Since $\bar{R}[U]$ is integral over $R[U], \mathfrak{p}_{U}$ is also maximal in $R[U]$ by (1.3). If we put $\mathfrak{p}^{\prime}=\mathfrak{p}_{U} R^{\prime}$, then we have

$$
R^{\prime} / \mathfrak{p}^{\prime} \cong\left(R[U] / \mathfrak{p}_{U}\right)\left[X_{2}, X_{3}, \cdots, X_{n}\right] .
$$

Obviously we have $\mathfrak{p}^{\prime} \subset \mathfrak{m}^{\prime}$. This completes our proof.

COROLlary 5.3. Let $R$ be a semi-local principal ideal domain and $R^{\prime}$ $=R\left[X_{1}, X_{2}, \cdots, X_{n}\right]$ be the polynomial ring with a finite number of variables $X_{1}, X_{2}, \cdots, X_{n}$ over $R$. Then any maximal ideal $\mathfrak{m}^{\prime}$ of $R^{\prime}$ is generated by elements in numbers equal to its height.

Now we give

THEOREM 5.4. Let $R$ be a semi-local integral domain of dimension 1 and let $R^{\prime}=R[X, Y]$ be the polynomial ring with two variables $X, Y$ over $R$. Then the following conditions are equivalent:

1) $R$ is a weakly normal ring.

2) $R^{\prime}$ is a PF domain.

Proof. As the implication 2$) \rightarrow 1$ ) was proved in (3.2), we have only to show the implication 1$) \rightarrow 2$ ). Suppose that $R$ is weakly normal. Let $\mathfrak{m}^{\prime}$ be a maximal ideal of height 2 in $R^{\prime}$. Then, by (5.2), there exists a prime ideal $\mathfrak{p}^{\prime}$ of height 1 in $R^{\prime}$ contained in $\mathrm{m}^{\prime}$ such that $R^{\prime} / \mathfrak{p}^{\prime}$ is isomorphic to the polynomial ring with a variable over a field, i. e., to a Euclidean ring. If we denote by $\mathfrak{n}$ the Jacobson radical of $R$, we see easily $\mathfrak{p}^{\prime}+\mathfrak{n} R^{\prime}=R^{\prime}$. Hence, by (3.1), $\mathfrak{p}^{\prime}$ is principal in $R^{\prime}$ and so we can put $\mathfrak{p}^{\prime}=p^{\prime} R^{\prime}$ for a prime element $p^{\prime}$ of $R^{\prime}$. Let $S^{\prime}$ be the multiplicative system of $R^{\prime}$ generated by all prime elements $\left\{p_{i}^{\prime}\right\}$ in $R^{\prime}$ such that any $R^{\prime} / p_{i}^{\prime} R^{\prime}$ is Euclidean. If a maximal ideal $\mathfrak{m}^{\prime}$ of $R^{\prime}$ has height 2 in $R^{\prime}$, then we have $\mathfrak{m}^{\prime} \cap S^{\prime} \neq \phi$ by the preceding argu- 
ment, and if $\mathfrak{m}^{\prime}$ has height 3 in $R^{\prime}$, then we have $\mathfrak{m}^{\prime} \cap S^{\prime}=\phi$, as is easily seen. If we put $R^{\prime \prime}=R_{S^{\prime}}^{\prime}$, there is no maximal ideal of height 2 in $R^{\prime \prime}$. Let $\mathfrak{m}^{\prime \prime}$ be a maximal ideal of height 1 in $R^{\prime \prime}$. If we put $\mathfrak{p}^{\prime}=\mathfrak{m}^{\prime \prime} \cap R^{\prime}$, then $\mathfrak{p}^{\prime}$ is a prime ideal of height 1 in $R^{\prime}$ such that $\mathfrak{p}^{\prime}+\mathfrak{n} R^{\prime}=R^{\prime}$. Again, by (3.1), $\mathfrak{p}^{\prime}$ is principal in $R^{\prime}$. As $\mathfrak{m}^{\prime \prime}=\mathfrak{p}^{\prime} R^{\prime \prime}, \mathfrak{m}^{\prime \prime}$ is also principal in $R^{\prime \prime}$. Let $S^{\prime \prime}$ be the multiplicative system of $R^{\prime \prime}$ generated by all prime elements $\left\{p_{i}^{\prime \prime}\right\}$ in $R^{\prime \prime}$ such that any $R^{\prime \prime} / p_{i}^{\prime \prime} R^{\prime \prime}$ is a field. Then, for a maximal ideal $\mathfrak{m}^{\prime \prime}$ of height 1 in $R^{\prime \prime}$, we have $\mathrm{m}^{\prime \prime} \cap S^{\prime \prime} \neq \phi$ by the preceding argument, and while, for a maximal ideal $\mathfrak{m}^{\prime \prime}$ of height 3 in $R^{\prime \prime}$, we have $\mathfrak{m}^{\prime \prime} \cap S^{\prime \prime}=\phi$. If we put $R^{*}=R_{S^{\prime \prime}}^{\prime \prime}$, then any maximal ideal $\mathfrak{m}^{*}$ of $R^{*}$ has height 3 in $R^{*}$ and is generated by a maximal ideal $\mathfrak{m}^{\prime}$ of height 3 in $R^{\prime}$. So we have $\mathfrak{m}^{*} \supset \mathfrak{n} R^{*}$. This shows that $\mathfrak{n} R^{*}$ is contained in the Jacobson radical of $R^{*}$. According to (2.2), if $R^{*}$ is a PF domain, then $R^{\prime \prime}$ is so and if $R^{\prime \prime}$ is a PF domain, then $R^{\prime}$ is so. Furthermore, if any finite projective $R^{*} / \mathrm{n} R^{*}$-module is quasi-free, then, by (2.5), $R^{*}$ is a PF domain. Therefore it suffices to prove that $R^{*} / \mathrm{n} R^{*}$ is a direct sum of PF domains. In fact, if we denote by $\mathfrak{m}_{1}, \mathfrak{m}_{2}, \cdots, \mathfrak{m}_{t}$ all maximal ideals of $R$, we have

$$
R^{*} / \mathfrak{n} R^{*} \cong(R / \mathfrak{n})[X, Y] \cong\left(R / \mathfrak{m}_{1}\right)[X, Y] \oplus \cdots \oplus\left(R / \mathfrak{m}_{t}\right)[X, Y] .
$$

As any $R / \mathfrak{m}_{i}$ is a field, any $\left(R / \mathfrak{m}_{i}\right)[X]$ is a principal ideal domain. Then, by (4.8), any $\left(R / \mathfrak{m}_{i}\right)[X, Y]$ is a PF domain. This completes our proof.

\section{Keiô University}

\section{References}

[1] H. Bass, Torsion-free and projective modules, Trans. Amer. Math. Soc., 102 (1962), 319-327.

[2] H. Bass and S. Schanuel, The homotopy theory of projective modules, Bull. Amer. Math. Soc., 68 (1962), 425-428.

[3] H. Cartan and S. Eilenberg, Homological algebra, Princeton Univ. Press, 1956.

[4] D. Lissner, Matrices over polynomial rings, Trans. Amer. Math. Soc., 98 (1961), 285-305.

[5] M. Nagata, Local rings, Interscience Publ., New York, 1962.

[6] J.P. Serre, Faisceaux algébrique cohérents, Ann. of Math., 61 (1955), 197-278.

[7] J.P. Serre, Modules projectifs et espaces fibrés à fibre vectorielle, Séminaire Dubreil-Pisot, t. 11, 1957-1958, n²3.

[8] J.P. Serre, Sur les modules projectifs, Séminaire Dubreil-Pisot, t. 14, 1960-1961, $n^{\circ} 2$.

[9] C.S. Seshadri, Triviality of vector bundles over the affine space $K^{2}$, Proc. Nat. Acad. Sci. U. S. A., 44 (1958), 456-458.

[10] C.S. Seshadri, Algebraic vector bundles over the product of an affine curve and an affine line, Proc. Amer. Math. Soc., 10 (1959), 670-673. 